\title{
基于绿色化学的有机化学实验室废弃物的原点处理
}

李培峰*, 高先池, 刘春颖

中国海洋大学化学化工学院, 山东青岛 266100

摘要: 有机化学实验是培养学生掌握实验基本技能和技术、提高动手能力的必修课。有机化学实验产生的废液成分 复杂, 尽管目前广大师生的环保意识比较强, 实验室废液能够做到分类收集并交由有资质的公司进行处理, 但是完 全依赖环保公司的处理成本比较高。因此我们尝试对有机化学实验室产生的废液进行实验室原点处理, 真正做到谁 污染、谁治理, 有效降低废液的处理成本, 并实现有机试剂的循环使用。

关键词: 绿色化学; 有机化学; 危险废弃物; 原点处理

中图分类号: G64; O6

\section{Origin Treatment of Organic Chemistry Laboratory Waste Based on Green Chemistry}

\section{Peifeng Li ${ }^{*}$, Xianchi Gao, Chunying Liu}

School of Chemical Engineering, Ocean University of China, Qingdao 266100, Shandong Province, China.

\begin{abstract}
Organic chemistry laboratory is a required course to cultivate students to master the basic lab skills and technology and improve their hands-on ability. Complex waste liquid was produced in the organic chemical laboratory. Although the environmental awareness of teachers and students is strong, laboratory waste liquid can be collected in the classification for processing by qualified companies. However, the cost is high, completely relying on environmental protection company processing. Then we try to treat the organic chemical laboratory waste liquid in the laboratory. The individual who produces waste must handle it. It can effectively reduce the treatment cost of waste liquid, and realize the recycling of organic reagents.
\end{abstract}

Key Words: Green chemistry; Organic chemistry; Laboratory dangerous waste; Origin treatment

有机化学实验是有机化学课程不可分割的教学内容, 是培养学生掌握实验基本技能和技术、提 高动手能力的必修课。有机化学实验中药品复杂, 产生的环境污染和能源消耗比较大。近年来, 政 府监管部门对高校实验室危险废弃物的监管力度逐渐增强。2005年7月26日, 我国教育部和国家环境 保护总局联合下发《关于加强高等学校实验室排污管理的通知》, 将高校实验室排污纳入环境监督 管理范围。随后, 国家不断出台各种相关的法规和标准进一步规范高校实验室危险废弃物的排放, 其中包括《废弃危险化学品污染环境防治法》《实验室危险废物污染防治技术规范DB11T1368-2016》 等。因此, 安全、规范、有效地对高校实验室危险废弃物进行管理成为高校实验室管理部门亟待解 决的问题。 


\section{1 高校化学实验室危险废弃物的管理现状}

随着高校招生规模的扩大和实验教学内容的改革, 学生人数和实验种类剧增, 对试剂的消耗也 随之增大, 产生废液的数量和种类也随之增加。这些废弃物渗入土壤、地下水, 或者飘入大气, 造 成环境污染, 最终影响人类健康。不同于化工企业产生的废液种类单一、单种废液数量大, 高校化 学实验室废液的特点是种类多, 每种废液的数量相对较少。但是如果处理不当, 也会造成安全事故, 对环境和人身造成伤害。近几年, 高校化学实验室安全事故频发, 因此, 高校作为教学和科研的重 要场所, 必须进一步加强实验室危险废弃物的收集与处置, 为社会输送具有安全与环保意识的大学 生。

继国家出台各种法规和标准后, 目前各大高校结合本校的实际情况, 逐步建立起实验室污染物 排放管理机制, 学校内部对实验室产生的危险废弃物从收集、存储、转运等方面进行规范化、精细 化管理, 基本都制定了相应的规章制度并不断完善 ${ }^{[1]}$ 。因此, 高校实验室危险废弃物的排放管理逐 渐走向正轨, 现在绝大多数高校实验室产生的危险废弃物基本都能做到分类回收和存放, 并将回收 后的废弃物交由有资质的环保公司进行处理。

\section{2 化学实验室废液的回收方法}

高校化学实验的危险废弃物主要包括固体废弃物、液体废弃物和气体废弃物。固体废弃物主要 包括空试剂瓶、碎玻璃、废弃包装物、被药品沾污的固体和过期的固体药品等。液体废弃物主要包 括无机废液、有机废液和过期药品。气体废弃物主要包括实验过程中产生的废气, 根据气体的性质 和浓度采取不同的处理方式, 高浓度的气体加装气体吸收装置进行处理, 低浓度的气体由排风装置 引入集中废气处理装置中进行处置, 然后排放到大气。固体和液体废弃物分类收集后交给有资质的 环保公司进行处理。这些废弃物中, 最难处理的是液体废弃物(废液)。废液的回收涉及到废液的分 类、存储和转运等, 不同的学校采取不同的废液分类方法 ${ }^{[2-4]}$ 。一般而言, 基础教学实验室的废液通 常分为无机废液和有机废液。无机废液包括含酸废液、含碱废液和重金属废液。有机废液则主要包 括含卤和不含卤的。在废液的回收过程中遵循 “专桶专用, 分类收集” 的原则, 回收桶上注明废液 的种类、主要成分、存放地点和应急联系人等信息, 并且在桶的下方放有托盘以防废液渗漏造成新 的污染。废液的收集登记需要建立台账, 必须做到有迹可循。随着信息技术的发展, 部分高校开发 建设了试剂使用平台, 从试剂的采购、使用到废液的回收等都可以在平台上进行记录, 需要进行回 收时在系统平台上提交申请即可, 这就使得废液的回收管理更加规范和方便 ${ }^{[5]}$ 。

\section{3 有机化学实验室废弃物的原点处理}

高校分类收集的废液需要交给有资质的环保公司进行无害化处理。一方面目前有资质的机构和 公司不多, 另一方面由于环保要求的提高和废液排放数量的增多, 环保公司废液处理能力不足的情 况逐渐显现, 甚至出现废液回收处理不畅通的局面。此外, 有资质的环保公司的收费标准较高, 高 校用于废液处理的费用也水涨船高, 这对于高校而言是一笔沉重的负担, 由于经费不足, 部分高校 在废液处理经费方面存在很大的缺口。因此, 高校不能仅仅满足于将危险废弃物分类收集后交给环 保公司, 而是应积极主动地寻找废弃物原点就近处理的新出路。但是目前国内高校在这方面的意识 还很淡薄，仅仅满足于废液的分类收集，尚未真正建立起谁排污谁负责的管理机制。

日本高校对实验室化学废液的管理起步较早, 形成了一套科学有效的管理机制。日本高校实验 室废弃物的管理主要坚持两项原则：一是 “排出者责任” 原则, 二是 “原点处理” 原则。“排出者 责任” 原则是指废液的排出者对废液的产生、收集、搬运直至最终的处理, 都负有相应的责任。

“原点处理” 原则是指尽量在废弃物产生的源头就近对其进行处理 ${ }^{[6]}$ 。这是因为排出者对废弃物的 成分和性质最清楚, 如果做到了原点处理, 将大大降低废液处理时间、人力、物力和成本, 也避免 了不同废液混合造成的新的污染和处理成本。因此, 日本高校在进行废液处理的过程中, 会优先考 
虑高校实验室内部来进行处理, 自己处理不了的废液交给环保公司处理。如日本的熊本大学是学校 处理有机废液, 委外处理无机废液 ${ }^{[6]}$ 。

有机化学实验是中国海洋大学化学化工专业、材料、生命科学、食品科学、水产和药学等近化 类专业的基础课程。自从我校实施《中国海洋大学实验室危险废弃物处置管理办法》以来, 有机化 学实验室产生的废液都能做到分类收集并交给有关环保公司进行处理。根据环保公司要求, 我们所 收集的有机废液必须集中到一个容器当中。在溶液的混合过程中很容易产生新的不安全隐患, 并 造成后续处理程序和成本的相应增加。另外, 目前我校完全依赖环保公司进行废弃物处理的专项支 出相对较高。因此, 我们结合有机化学实验室产生的有机废弃物的成分和性质, 借鉴日本高校实验 室废弃物的处理方法, 尝试有机化学实验室废弃物的原点处理, 进行绿色化学理念在有机化学实验 室废弃物管理方面的探索, 真正做到谁污染, 谁处理。

有机化学实验室产生的废液主要包括大量的有机废液( $99 \%$ 以上)和少量的无机废液(1\%左 右)。含酸或者含碱的无机废液可以通过用废酸废碱进行中和的方式处理, 通过测定溶液的 $\mathrm{pH}$, 达标 后再稀释排放。而实验室产生的有机废液成分比较复杂, 需要根据废液的浓度、性质进行分类处理, 这部分废液是有机实验室原点处理的难点和重点。以中国海洋大学鱼山校区的两个实验室为例, 我 们进行了有机废液原点处理的尝试。这两个实验室主要针对食品、生命、水产等专业开放, 48学时, 每年选课人数约 500 人左右, 主要开设的实验课程包括蒸馏与分馏、水蒸气蒸馏、重结晶及过滤、熔 点的测定、正澳丁烷的制备、乙酸正丁酯的制备、乙酰苯胺的制备、从茶叶中提取咖啡因等。蒸馏 与分馏、水蒸气蒸馏和从茶叶中提取咖啡因这三个实验回收的废液主要是浓度比较高的实验试剂, 分别是丙酮、乙酸异戊酯和乙醇。正澳丁烷的制备和乙酸正丁酯的制备这两个制备实验收集的废液 主要是学生实验的产物, 分别是正溴丁烷和乙酸正丁酯。上述实验回收的大部分废液, 浓度比较高, 成分单一, 直接作为废液交给环保公司处理掉非常可惜, 因此, 我们通过采取蒸馏、萃取和干燥等 简单操作就可以进行实验室提纯, 提纯后的丙酮、乙酸异戊酯和乙醇继续用于以后的学生实验。以 含丙酮的废液为例, 由于这个实验是学生进行蒸馏操作的第一个实验, 因此收集的产物纯度不高, 我们将所有学生回收的丙酮收集在一起, 重新进行蒸馏, 即可得到纯度比较高的丙酮。同样, 乙酰 苯胺的制备实验中收集的固体乙酰苯胺, 可以直接用于熔点的测定实验的原料, 也可以添加少量杂 质用于重结晶及过滤实验的原料, 实现了试剂的循环使用。

通过有机化学实验室废液的原点处理, 减少了实验室废弃物的处理环节, 同时大大降低了废弃 物在咜存、运输和处理过程中的安全隐患及带来的环境污染。将处理提纯后的药品变废为宝, 循环 使用, 继续用于学生实验, 使得有机化学实验室的试剂成本大大降低, 初步估计鱼山校区的两个有 机化学实验室中, 丙酮、乙酸异戊酯、乙醇、乙酰苯胺等试剂消耗可以节约 $50 \%$ 左右。试剂用量的减 少, 使得试剂的订购、存咜和安全管理也变得简单。另外, 废液一经产生马上进行处理, 废液数量 比较少, 实验室处理起来相对简单, 不会增加很多工作量, 对于实验教师而言不会造成很大负担。 近期我们正在尝试吸引学生参与, 通过采取开放实验的方式鼓励课堂掌握不好的学生以及需要增加 社会实践的学生参与进来, 进一步培养学生的环保意识和动手操作能力, 可谓一举多得。

\section{4 结语}

高校化学实验室危险废弃物的管理是一项系统工程, 上至国家、下至院校, 正在加强进行危险 废弃物排放管理的制度和体制建设, 高校实验室危险废弃物排放的法律法规、政策措施和管理细则 正在日益完善。我们尝试从绿色化学的角度进行有机化学实验室危险废弃物的原点处理, 目前工作 取得了一定的成效, 但是也还存在很多问题, 需要在后期的工作中慢慢摸索。我们希望以此为切入 点进行基础教学化学实验室其他废弃物的原点处理, 力争最大限度地将污染控制在源头处。同时, 这项工作需要学校在专项经费和政策支持方面的适当倾斜, 鼓励实验技术人员和专职教师利用专业 知识进行实验室废弃物的原点处理。 
[1] 彭实, 田曙坚, 吴良莉, 刘济昌. 环境保护科学, 2009, 35 (6), 14.

[2] 姜雨生, 谢凯, 唐丹平, 田巍, 肖晓峰, 高晓颖. 环境工程技术学报, 2016, 6 (5), 447.

[3] 兰景风, 俞娥. 大学化学, 2016, 31 (8), 71 .

[4] 王岩, 彭实, 田曙坚. 实验技术与管理, 2009, 26 (8), 179.

[5] 常静, 商闯. 大学化学, 2021, 36 (2), 2004032.

[6] 彭实, 沈立娜, 吴良莉. 实验技术与管理, 2009, 26 (6), 165. 